\title{
Grid classes and the Fibonacci dichotomy for restricted permutations
}

\author{
Sophie Huczynska* and Vincent Vatter ${ }^{\dagger}$ \\ School of Mathematics and Statistics \\ University of St Andrews \\ St Andrews, Fife, Scotland \\ \{sophieh, vince\}@mcs.st-and.ac.uk \\ http://turnbull.mcs.st-and.ac.uk/ $\sim$ sophieh, vince $\}$
}

Submitted: Feb 9, 2006; Accepted: Jun 5, 2006; Published: Jun 23, 2006

Mathematics Subject Classification: 05A05, 05A15, 05A16

\begin{abstract}
We introduce and characterise grid classes, which are natural generalisations of other well-studied permutation classes. This characterisation allows us to give a new, short proof of the Fibonacci dichotomy: the number of permutations of length $n$ in a permutation class is either at least as large as the $n$th Fibonacci number or is eventually polynomial.
\end{abstract}

\section{Introduction}

A permutation $\pi$ of $[n]^{1}$ contains the permutation $\sigma$ of $[k](\sigma \leq \pi)$ if $\pi$ has a subsequence of length $k$ in the same relative order as $\sigma$. For example, $\pi=391867452$ (written in list, or one-line notation) contains $\sigma=51342$, as can be seen by considering the subsequence $91672(=\pi(2), \pi(3), \pi(5), \pi(6), \pi(9))$. A permutation class is a downset of permutations under this order, or in other words, if $\mathcal{C}$ is a permutation class, $\pi \in \mathcal{C}$, and $\sigma \leq \pi$, then $\sigma \in \mathcal{C}$. We shall denote by $\mathcal{C}_{n}(n \in \mathbb{N})$ the set $\mathcal{C} \cap S_{n}$, i.e. those permutations in $\mathcal{C}$ of length $n$. Recall that an antichain is a set of pairwise incomparable elements. For any permutation class $\mathcal{C}$, there is a unique (and possibly infinite) antichain $B$ such that $\mathcal{C}=\operatorname{Av}(B)=\{\pi: \beta \not \leq \pi$ for all $\beta \in B\}$. This antichain $B$ is called the basis of $\mathcal{C}$. Permutation classes arise naturally in a variety of disparate fields, ranging from the analysis of sorting machines (dating back to Knuth [13], who proved that a permutation is

\footnotetext{
*Supported by a Royal Society Dorothy Hodgkin Research Fellowship.

†'Supported by EPSRC grant GR/S53503/01.

${ }^{1}$ Here $[n]=\{1,2, \ldots, n\}$ and, more generally, for $a, b \in \mathbb{N}(a<b)$, the interval $\{a, a+1, \ldots, b\}$ is denoted by $[a, b]$, the interval $\{a+1, a+2, \ldots, b\}$ is denoted by $(a, b]$, and so on.
} 


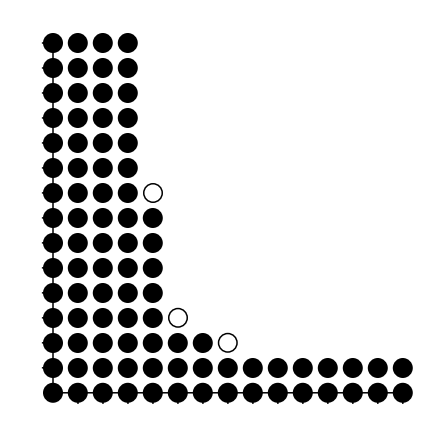

Figure 1: The plot of downset in $\mathbb{N}^{2}$; the elements of the class are drawn with solid circles, while the elements of the basis are drawn with hollow circles.

stack-sortable if and only if it lies in the class $\operatorname{Av}(231))$ to the study of Schubert varieties (see, e.g., Lakshmibai and Sandhya [14]).

The Stanley-Wilf Conjecture, recently proved by Markus and Tardos [15], states that all permutation classes except the set of all permutations have at most exponential growth, i.e., for every class $\mathcal{C}$ with a nonempty basis, there is a constant $K$ so that $\left|\mathcal{C}_{n}\right|<K^{n}$ for all $n$. Less is known regarding the exact enumeration of permutation classes. Natural enumerative questions include:

(i) Which permutation classes are finite?

(ii) Which permutation classes are enumerated by a polynomial?

(iii) Which permutation classes have rational generating functions? (We refer to $\sum\left|\mathcal{C}_{n}\right| x^{n}$ as the generating function of $\mathcal{C}$.)

(iv) Which permutation classes have algebraic generating functions?

(v) Which permutation classes have $P$-recursive enumeration?

The answer to the first question on this list follows easily from the Erdős-Szekeres Theo$\mathrm{rem}^{2}$ : the class $\operatorname{Av}(B)$ is finite if and only if $B$ contains both an increasing permutation and a decreasing permutation. The answer to the second question is provided in this paper. Questions (iii)-(v) remain unanswered.

Downsets of vectors. Perhaps the simplest interesting context in which to study downsets is finite vectors of nonnegative integers, and in this context there is also a polynomial enumeration result which we shall employ in our proofs. Let $\mathbf{x}=\left(x_{1}, \ldots, x_{m}\right), \mathbf{y}=$ $\left(y_{1}, \ldots, y_{m}\right) \in \mathbb{N}^{m}$ for some $m$. We write $\mathbf{x} \leq \mathbf{y}$ if $x_{i} \leq y_{i}$ for all $i \in[m]$. This order is often called the product order. The weight of the vector $\mathbf{x}$, denoted $\|\mathbf{x}\|$, is the sum of the entries of $\mathbf{x}$.

\footnotetext{
${ }^{2}$ The Erdős-Szekeres Theorem [9]. Every permutation of length $n$ contains a monotone subsequence of length at least $\sqrt{n}$.
} 


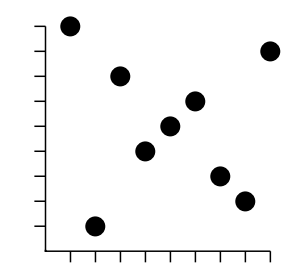

Figure 2: The plot of the skew-merged permutation 917456328.

Theorem 1.1. Let $\mathcal{C}$ denote a downset in $\mathbb{N}^{m}$. For sufficiently large $n$, the number of vectors in $\mathcal{C}$ of weight $n$ is given by a polynomial.

Stanley [20] posed Theorem 1.1 as a Monthly problem in 1976 and offered two solutions. One of these solutions is elementary while the other follows from viewing the number of vectors in question as a Hilbert function.

Downsets of other objects. Downsets of other combinatorial objects have been extensively studied, and other polynomial enumeration results are known. These have often been established by ideas analogous to the grid classes of matchings we use.

For example, downsets of graphs with respect to the induced subgraph ordering that are closed under isomorphism are called hereditary properties. Let $\mathcal{P}$ denote a hereditary property, and let $\mathcal{P}_{n}$ denote the set of graphs in $\mathcal{P}$ with vertex set $[n]$. Scheinerman and Zito [18] proved that $\left|\mathcal{P}_{n}\right|$ either has polynomial growth (meaning that $\left|\mathcal{P}_{n}\right|=\Theta\left(n^{k}\right)$ for some $k$ ) or $\left|\mathcal{P}_{n}\right|$ has at least exponential growth. Balogh, Bollobás, and Weinreich [8] later showed that polynomial growth hereditary properties are enumerated exactly by a polynomial for large $n$. Their proof of this result uses "canonical properties," which are quite like our grid classes of matchings.

Moving to a more general context, Pouzet and Thiéry [17] study polynomial growth (although not exact polynomial enumeration) for certain downsets of relational structures. While summarising their work would take us too far afield, we remark first that permutations can be viewed as relational structures ${ }^{3}$ and second that the grid classes of matchings we use essentially correspond to their concept of "monomorphic decompositions into finitely many parts."

\section{Grid classes}

\subsection{The skew-merged permutations}

We begin with an example of a grid class. A permutation is said to be skew-merged if it is the union of an increasing subsequence and a decreasing subsequence. For example, the permutation shown in Figure 2 is skew-merged. Stankova [19] was the first to find the

\footnotetext{
${ }^{3}$ E.g., $\pi \in S_{n}$ can be taken to correspond to the relational structure on $[n]$ with two linear orders, $<$ and $\prec$, where $<$ is the normal ordering of $[n]$ and $i \prec j \Longleftrightarrow \pi(i)<\pi(j)$.
} 
basis of this class. Later, Kézdy, Snevily, and Wang [12] observed that the basis follows easily from Földes and Hammer's characterisation of split graphs ${ }^{4}$ in [10].

Theorem 2.1 (Stankova [19]; Kézdy, Snevily, and Wang [12]; and Atkinson [5]). The skew-merged permutations are $\operatorname{Av}(2143,3412)$.

Atkinson [5] showed that the generating function for the skew-merged permutations is given by

$$
\frac{1-3 x}{(1-2 x) \sqrt{1-4 x}} .
$$

Kézdy, Snevily, and Wang [12] studied one generalization of skew-merged permutations, the class of permutations which can be partitioned into $r$ increasing subsequences and $s$ decreasing subsequences. Grid classes provide a different generalization.

\subsection{Definitions}

First an important warning: when discussing grid classes, we index matrices beginning from the lower left-hand corner, and we reverse the rows and columns; for example $M_{3,2}$ denotes for us the entry of $M$ in the 3rd column from the left and 2nd row from the bottom. Below we include a matrix with its entries labeled:

$$
\left(\begin{array}{lll}
(1,2) & (2,2) & (3,2) \\
(1,1) & (2,1) & (3,1)
\end{array}\right) .
$$

Roughly, the grid class of a matrix $M$ is the set of all permutations that can be divided in a prescribed manner (dictated by $M$ ) into a finite number of blocks, each containing a monotone subsequence. We have already introduced the best-studied grid class, the skew-merged permutations. We previously defined them as the permutations that can be written as the union of an increasing subsequence and a decreasing subsequence. As a grid class, the skew-merged permutations can be defined as the permutations that can be divided into four monotonic blocks, two increasing and two decreasing, as indicated in Figure 3, and our notation for this class is

$$
\operatorname{Grid}\left(\begin{array}{rr}
-1 & 1 \\
1 & -1
\end{array}\right) \text {, }
$$

but before reaching that point we need to introduce some notation.

Given a permutation $\pi \in S_{n}$ and sets $A, B \subseteq[n]$, we write $\pi(A \times B)$ for the subsequence of $\pi$ with indices from $A$ which has values in $B$. For example, applying this operation to the permutation shown in Figure 3, we get

$$
917456328([5] \times[5])=1,4,5,
$$

\footnotetext{
${ }^{4} \mathrm{~A}$ graph $G$ is split if its vertices can be partitioned into a disjoint union $V(G)=V_{1} \uplus V_{2}$ s.t. $G\left[V_{1}\right]$ is complete and $G\left[V_{2}\right]$ is edgeless. Földes and Hammer proved that a graph is split if and only if it does not contain $K_{2} \uplus K_{2}, C_{4}$, or $C_{5}$ as induced subgraphs.
} 


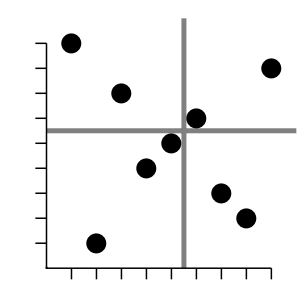

Figure 3: A gridding of the skew-merged permutation 917456328.

and this (increasing) subsequence gives the points in the lower left-hand box of Figure 3 . The increasing subsequence in the upper right-hand box is

$$
917456328([6,9] \times[6,9])=6,8
$$

while the decreasing subsequence in the lower right-hand box is

$$
917456328([6,9] \times[5])=3,2 .
$$

Now suppose that $M$ is a $t \times u$ matrix (meaning, in the notation of this paper, that it has $t$ columns and $u$ rows). An $M$-gridding of the permutation $\pi \in S_{n}$ is a pair of sequences $1=c_{1} \leq \cdots \leq c_{t+1}=n+1$ (the column divisions) and $1=r_{1} \leq \cdots \leq r_{u+1}=n+1$ (the row divisions) such that for all $k \in[t]$ and $\ell \in[u], \pi\left(\left[c_{k}, c_{k+1}\right) \times\left[r_{\ell}, r_{\ell+1}\right)\right)$ is:

- increasing if $M_{k, \ell}=1$,

- decreasing if $M_{k, \ell}=-1$,

- empty if $M_{k, \ell}=0$.

We define the grid class of $M$, written $\operatorname{Grid}(M)$, to be the set of all permutations that possess an $M$-gridding. We say that $\pi$ is $t \times u$-griddable if it is $M$-griddable for some $t \times u$ matrix $M$.

A class $\mathcal{C}$ is said to be $t \times u$-griddable if every permutation in $\mathcal{C}$ is $t \times u$-griddable, and it is said to be griddable if it is $t \times u$-griddable for some $t, u \in \mathbb{N}$. Note that all griddable classes lie in some particular grid class (suppose that $\mathcal{C}$ is $t \times u$ griddable and take a larger matrix $M$ containing every $t \times u$ matrix, then $\mathcal{C}$ lies in $\operatorname{Grid}(M))$.

Two special types of grid classes have been extensively studied. One type is the profile classes of Atkinson [6], which in our language are grid classes of permutation matrices. Another example of grid classes are the $W$-classes introduced by Atkinson, Murphy, and Ruškuc [7], which are the grid classes of $0 / \pm 1$ row vectors.

Atkinson, Murphy, and Ruškuc [7] introduced $W$-classes in their study of partially well-ordered (pwo $)^{5}$ permutation classes, and proved that grid classes of $0 / \pm 1$ row vectors are pwo. This result does not extend to arbitrary grid classes, i.e., some grid classes contain

\footnotetext{
${ }^{5}$ Recall that a partially ordered set is said to be partially well-ordered (pwo) if it contains neither an infinite properly decreasing sequence nor an infinite antichain.
} 
infinite antichains, e.g., there is an infinite antichain of skew-merged permutations. In order to characterise the pwo grid classes, we associate a graph to each grid class. For any $t \times u$ matrix $M$ we construct the bipartite graph $G(M)$ with vertices $x_{1}, \ldots, x_{t}$ and $y_{1}, \ldots, y_{u}$ and edges $x_{k} y_{\ell}$ precisely when $M_{k, \ell} \neq 0$. For example, the bipartite graph of a vector is a star together with isolated vertices, while the bipartite graph of $\left(\begin{array}{rr}-1 & 1 \\ 1 & -1\end{array}\right)$ is a cycle with 4 vertices. The pwo properties of a grid class depend only on its graph.

Theorem 2.2 (Murphy and Vatter [16]). The grid class of $M$ is pwo if and only if $G(M)$ is a forest.

\subsection{The characterisation of griddable classes}

It appears surprisingly difficult to compute the basis of $\operatorname{Grid}(M)$ when $M$ is neither a vector nor a permutation matrix. Waton [private communication] has computed the bases of $\operatorname{Grid}(M)$ for all $2 \times 2$ matrices $M$, but we know of no such results for larger matrices. In particular, the following remains a conjecture.

Conjecture 2.3. All grid classes are finitely based.

We instead take a coarser approach and ask only for a characterisation of the griddable classes, that is, the permutation classes that lie in some grid class.

It will prove useful to have an alternative interpretation of griddability. We say that the permutation $\pi \in S_{n}$ can be covered by $s$ monotonic rectangles if there are $\left[w_{1}, x_{1}\right] \times$ $\left[y_{1}, z_{1}\right], \ldots,\left[w_{s}, x_{s}\right] \times\left[y_{s}, z_{s}\right] \subseteq[n] \times[n]$ such that

- for each $i \in[s], \pi\left(\left[w_{i}, x_{i}\right] \times\left[y_{i}, z_{i}\right]\right)$ is monotone, and

- $\bigcup_{i \in[s]}\left[w_{i}, x_{i}\right] \times\left[y_{i}, z_{i}\right]=[n] \times[n]$.

Note that we allow these rectangles to intersect. By definition every $t \times u$-griddable permutation can be covered by $t u$ monotonic rectangles. The following proposition gives the other direction.

Proposition 2.4. Every permutation that may be covered by $s$ monotonic rectangles is $(2 s-1) \times(2 s-1)$-griddable.

Proof. Suppose that $\pi \in S_{n}$ is covered by the $s$ monotonic rectangles $\left[w_{1}, x_{1}\right] \times\left[y_{1}, z_{1}\right]$, $\ldots,\left[w_{s}, x_{s}\right] \times\left[y_{s}, z_{s}\right] \subseteq[n] \times[n]$. Define the indices $c_{1}, \ldots, c_{2 s}$ and $r_{1}, \ldots, r_{2 s}$ by

$$
\begin{aligned}
& \left\{c_{1} \leq \cdots \leq c_{2 s}\right\}=\left\{w_{1}, x_{1}, \ldots, w_{s}, x_{s}\right\} \\
& \left\{r_{1} \leq \cdots \leq r_{2 s}\right\}=\left\{y_{1}, z_{1}, \ldots, y_{s}, z_{s}\right\}
\end{aligned}
$$

Since these rectangles cover $\pi$, we must have $c_{1}=r_{1}=1$ and $c_{2 s}=r_{2 s}=n$. Now we claim that these sets of indices form an $M$-gridding of $\pi$ for some $2 s-1 \times 2 s-1$ matrix $M$. 


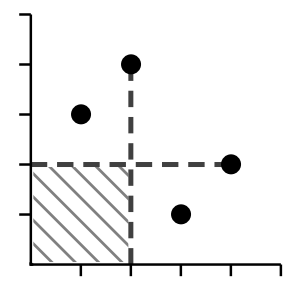

(i)

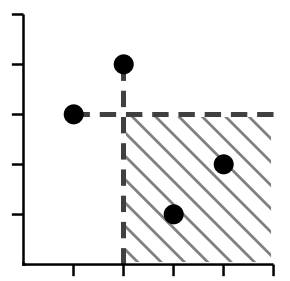

(ii)

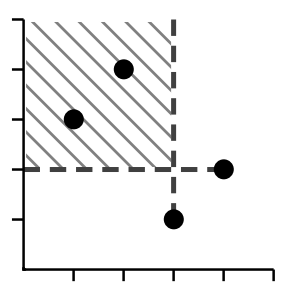

(iii)

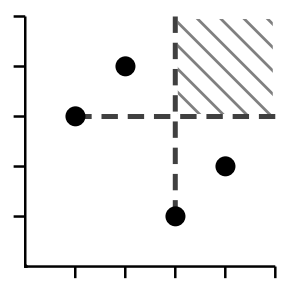

(iv)

Figure 4: The regions of $\pi$ referred to in the proof of Theorem 2.5.

To prove this claim it suffices to show that $\pi\left(\left[c_{k}, c_{k+1}\right] \times\left[r_{\ell}, r_{\ell+1}\right]\right)$ is monotone for every $k, \ell \in[2 s-1]$, since we can then construct the matrix $M$ based on whether this subsequence is increasing or decreasing. Because the rectangles given cover $\pi$, the point $\left(c_{k}, r_{\ell}\right)$ lies in at least one rectangle, say $\left[w_{m}, x_{m}\right] \times\left[y_{m}, z_{m}\right]$. Thus $c_{k} \geq w_{m}$ and $r_{\ell} \geq y_{m}$ and, because of the ordering of the $c$ 's and $r$ 's, we have $c_{k+1} \leq x_{m}$ and $r_{\ell+1} \leq z_{m}$. Therefore $\left[c_{k}, c_{k+1}\right] \times\left[r_{\ell}, r_{\ell+1}\right]$ is contained in $\left[w_{m}, x_{m}\right] \times\left[y_{m}, z_{m}\right]$ and so $\pi\left(\left[c_{k}, c_{k+1}\right] \times\right.$ $\left.\left[r_{\ell}, r_{\ell+1}\right]\right)$ is monotone.

With this new interpretation of griddability established, we need only two more definitions before characterising the griddable classes. Given two permutations $\pi \in S_{m}$ and $\sigma \in S_{n}$, we define their direct sum, written $\pi \oplus \sigma$ by

$$
(\pi \oplus \sigma)(i)= \begin{cases}\pi(i) & \text { if } i \in[m], \\ \sigma(i-m)+m & \text { if } i \in[m+n] \backslash[m],\end{cases}
$$

and similarly define their skew sum by

$$
(\pi \ominus \sigma)(i)= \begin{cases}\pi(i)+n & \text { if } i \in[m], \\ \sigma(i-m) & \text { if } i \in[m+n] \backslash[m] .\end{cases}
$$

Theorem 2.5. A permutation class is griddable if and only if it does not contain arbitrarily long direct sums of 21 or skew sums of 12 .

Proof. If a permutation class does contain arbitrarily long direct sums of 21 or skew sums of 12 , then it is clearly not griddable.

For the other direction, let $\mathcal{C}$ be a permutation class that does not contain $\ominus^{a+1} 12$ or $\oplus^{b+1} 21$. We show by induction on $a+b$ that there is a function $f(a, b)$ so that every permutation in $\mathcal{C}$ can be covered by $f(a, b)$ monotonic rectangles, and thus we will be done by Proposition 2.4 .

First note that if either $a$ or $b$ is 0 then $\mathcal{C}$ can only contain monotone permutations, so we can set $f(a, 0)=f(0, b)=1$. The next case is $a+b=2$, and since we may assume that $a, b \neq 0$, we have $a=b=1$. Thus $\mathcal{C}$ contains neither $\ominus^{2} 12=3412$ nor $\oplus^{2} 21=2143$, so $\mathcal{C}$ is a subclass of the skew-merged permutations and thus every permutation in $\mathcal{C}$ may be covered by 4 monotonic rectangles and we may take $f(1,1)=4$.

By symmetry and the cases we have already handled, we may assume that $a \geq 2$ and $b \geq 1$. Let $\pi \in \mathcal{C}_{n}$ be a 3412-containing permutation (if there are no such permutations, 
then we are done by induction) and suppose that $\pi\left(i_{1}\right) \pi\left(i_{2}\right) \pi\left(i_{3}\right) \pi\left(i_{4}\right)$ is in the same relative order as 3412 where $1 \leq i_{1}<i_{2}<i_{3}<i_{4} \leq n$. By induction we have the following (see Figure 4 for an illustration of these regions):

(i) $\pi\left(\left[i_{2}\right] \times\left[\pi\left(i_{4}\right)\right]\right)$ avoids $\ominus^{a+1} 12$ and $\oplus^{b} 21$ so it can be covered by $f(a, b-1)$ monotonic rectangles,

(ii) $\pi\left(\left[i_{2}, n\right] \times\left[\pi\left(i_{1}\right)\right]\right)$ avoids $\ominus^{a} 12$ and $\oplus^{b+1} 21$ so it can be covered by $f(a-1, b)$ monotonic rectangles,

(iii) $\pi\left(\left[i_{3}\right] \times\left[\pi\left(i_{4}\right), n\right]\right)$ avoids $\ominus^{a} 12$ and $\oplus^{b+1} 21$ so it can be covered by $f(a-1, b)$ monotonic rectangles, and

(iv) $\pi\left(\left[i_{3}, n\right] \times\left[\pi\left(i_{1}\right), n\right]\right)$ avoids $\ominus^{a+1} 12$ and $\oplus^{b} 21$ so it can be covered by $f(a, b-1)$ monotonic rectangles.

Because the four regions in (i)-(iv) cover $\pi$, it may be covered by $2 f(a-1, b)+2 f(a, b-1)$ monotonic rectangles. Furthermore, the 3412-avoiding permutations in $\mathcal{C}$ may be covered by $f(1, b) \leq f(a-1, b)$ monotonic rectangles by induction, so we may take $f(a, b)=$ $2 f(a-1, b)+2 f(a, b-1)$, completing the proof.

\subsection{The enumeration of grid classes}

To date only scattered results are known about the enumeration of grid classes and their subclasses. The only general results are the following two.

Theorem 2.6 (Atkinson [6]). If $M$ is a permutation matrix, then $\operatorname{Grid}(M)$ and all its subclasses have eventually polynomial enumeration.

Theorem 2.7 (Albert, Atkinson, and Ruškuc [3]). If $G(M)$ is a star, then $\operatorname{Grid}(M)$ and all its subclasses have rational (and readily computable) generating functions.

It is very tempting to speculate that the enumerative properties of a grid class depend only on its graph ${ }^{6}$. Our contribution to this suspicion is to show (in Theorem 2.9) that when $G(M)$ is a matching ${ }^{7}$ then $\operatorname{Grid}(M)$ and all its subclasses have eventually polynomial enumeration, thus generalising Theorem 2.6. For brevity, we refer to such classes as the grid classes of matchings.

Theorem 2.9. If the permutation class $\mathcal{C}$ lies in the grid class of a matching then there is a polynomial $p(n)$ so that $\left|\mathcal{C}_{n}\right|=p(n)$ for all sufficiently large $n$.

\footnotetext{
${ }^{6}$ For example:

Conjecture 2.8. If $G(M)$ is a forest then $\operatorname{Grid}(M)$ and all its subclasses have rational generating functions.
}

${ }^{7}$ We take a matching to be a graph without incident edges, i.e., a graph with maximum degree 1. 


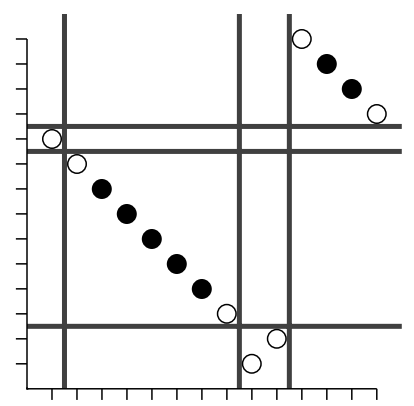

Figure 5: A greedy gridding of a permutation, showing its peg points as hollow circles; the peg permutation for this permutation is 5431276 while its non-peg vector is $(0,5,0,2)$. Note that since this is a greedy gridding, the $(1,3)$ entry of the corresponding matrix must be 1 .

Proof. Let $M$ be a $t \times u$ matrix whose graph is a matching, let $\mathcal{C}$ be a subclass of $\operatorname{Grid}(M)$, and let $\pi \in \mathcal{C}$. We define the greedy $M$-gridding of $\pi$ to be the gridding given by $1=$ $c_{1} \leq \cdots \leq c_{t+1}=n+1$ (the column divisions) and $1=r_{1} \leq \cdots \leq r_{u+1}=n+1$ (the row divisions) where for each $k, c_{k}$ is chosen so as to maximise $c_{1}+\cdots+c_{k}$. Because $G(M)$ is a matching, this uniquely defines the $r$ 's.

We define a peg point of $\pi$ to be a point which is either first or last (either horizontally or vertically; since the blocks are monotone, it doesn't matter) in its block in the greedy $M$-gridding of $\pi$. An example is shown in Figure 5. The peg permutation, $\rho^{\pi}$, of $\pi$ is then the permutation formed by its peg points. We also associate to each permutation $\pi \in \mathcal{C}$ its non-peg vector $\mathbf{y}^{\pi}=\left(y_{1}, \ldots, y_{t}\right)$, where $y_{i}$ denotes the number of non-peg points in $\pi\left(\left[c_{i}, c_{i+1}\right) \times[n]\right)$. Because the $M$-gridding was chosen greedily, the pair $\left(\rho^{\pi}, \mathbf{y}^{\pi}\right)$ uniquely determines $\pi$.

We now partition the class $\mathcal{C}$ based upon peg permutations. Since there can be at most $3^{t}$ different peg permutations of members of $\mathcal{C}$ (for every column of $M$ a peg permutation can have 0,1 , or 2 elements), this is a partition into a finite number of subsets. Let $\mathcal{C}^{\rho}$ denote the subset of $\mathcal{C}$ with peg permutation $\rho$. This is not a permutation class (the peg permutation of $\sigma \leq \pi$ need not be the peg permutation of $\pi$ ), but the set of nonpeg vectors of permutations in this class, $\left\{\mathbf{y}^{\pi}: \pi \in \mathcal{C}^{\rho}\right\}$, is a downset of vectors in $\mathbb{N}^{t}$. Therefore Theorem 1.1 shows that $\mathcal{C}^{\rho}$ has eventually polynomial enumeration, and so $\mathcal{C}$ does as well.

\section{The Fibonacci dichotomy}

The Fibonacci dichotomy for permutation classes, first proved by Kaiser and Klazar [11], states that all sub-Fibonacci permutation classes $^{8}$ have eventually polynomial enumeration. Here we give a new proof using the characterisation of grid classes. We have already

\footnotetext{
${ }^{8}$ We call a class $\mathcal{C}$ sub-Fibonacci if $\left|\mathcal{C}_{n}\right|$ is strictly less than the $n$th Fibonacci number for some $n$. The definition of sub- $2^{n-1}$ is analogous.
} 

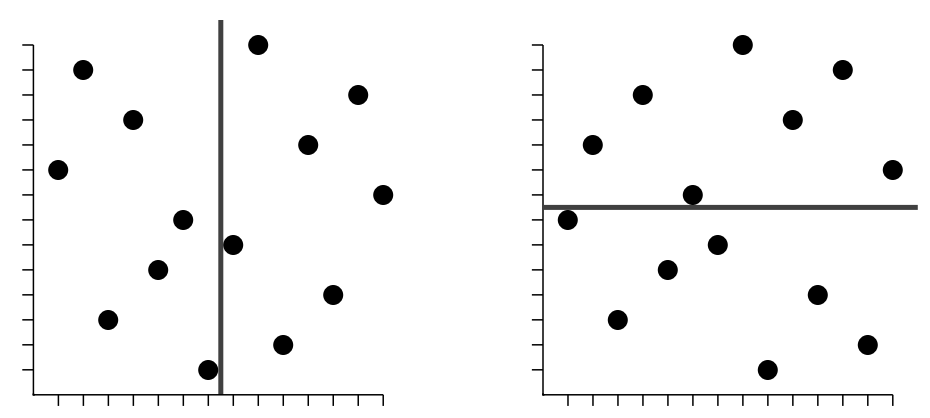

Figure 6: A horizontal alternation (left) and its inverse, a vertical alternation (right).

shown, in Theorem 2.9, that grid classes of matchings and their subclasses have eventually polynomial enumeration. It remains only to show that all sub-Fibonacci classes lie in grid classes of matchings. We do this in two parts. First we observe in Proposition 3.1 that all sub-Fibonacci classes are griddable, and then we show in Proposition 3.3 that all sub- $2^{n-1}$ griddable classes (which includes sub-Fibonacci classes) lie in grid classes of matchings.

Proposition 3.1. All sub-Fibonacci classes are griddable.

Proof. Let $\mathcal{C}$ denote a non-griddable class, so by Theorem 2.5 and symmetry we may assume that $\mathcal{C}$ contains arbitrarily long direct sums of 21. Since $\mathcal{C}$ is a permutation class, it must also contain every permutation that embeds into an arbitarily long direct sum of 21. These permutations have the form $\sigma_{1} \oplus \cdots \oplus \sigma_{k}$ where each $\sigma_{i}$ is either 1 or 21 . Thus there are precisely as many permutations of this form of length $n$ as there are ways of writing $n$ as an ordered sum of 1's and 2's, of which there are $F_{n}$.

A horizontal alternation is a permutation in which every odd entry lies to the left of every even entry, or the reverse of such a permutation. A vertical alternation is the group-theoretic inverse of a horizontal alternation. Examples are shown in Figure 6. We begin by observing that classes with arbitrarily long alternations are not small.

Proposition 3.2. If the permutation class $\mathcal{C}$ contains arbitrarily long alternations, then $\left|\mathcal{C}_{n}\right| \geq 2^{n-1}$ for all $n$.

Proof. By symmetry, let us suppose that $\mathcal{C}$ contains arbitrarily long horizontal alternations. By the Erdős-Szekeres Theorem, $\mathcal{C}$ contains arbitrarily long horizontal alternations in which both sides are monotone. Therefore $\mathcal{C}$ contains either $\operatorname{Grid}(11), \operatorname{Grid}(1-1)$, $\operatorname{Grid}(-11)$, or $\operatorname{Grid}(-1-1)$. It is easy to compute that the first and last of these classes contain $2^{n}-n$ permutations of length $n$ for $n \geq 1$ while the second and third contain $2^{n-1}$ permutations of length $n \geq 1$, establishing the proposition.

Therefore a sub-Fibonacci class cannot contain arbitrarily long alternations. We now prove that this implies that these classes lie in grid classes of matchings.

We say that a list of indices $i_{1}, \ldots, i_{s}$ in $\pi$ is an uninterrupted monotone interval if $\left|i_{j+1}-i_{j}\right|=1$ and $\left|\pi\left(i_{j+1}\right)-\pi\left(i_{j}\right)\right|=1$ for all $j \in[s-1]$. Note that if $G(M)$ is a matching, 
then an $M$-gridding of $\pi$ is a division of the elements of $\pi$ into uninterrupted monotone intervals. Conversely, every division of $\pi$ into uninterrupted monotone intervals gives an $M$-gridding of $\pi$ for some $M$ where $G(M)$ is a matching.

Proposition 3.3. A griddable class lies in the grid class of a matching if and only if it does not contain arbitrarily long alternations.

Proof. One direction is obvious: if a permutation class contains arbitrarily long alternations then it cannot lie in the grid class of a matching. The other direction is almost as clear, but a formal proof takes a modest amount of effort.

Let $\mathcal{C} \subseteq \operatorname{Grid}(N)$ for some $t \times u$ matrix $N$, and suppose that $\mathcal{C}$ does not contain any alternations (either horizontal or vertical) with more than $d$ elements. It suffices to show that there is a constant $m$ such that every permutation $\pi \in \mathcal{C}$ lies in $\operatorname{Grid}(M)$ where $G(M)$ is a matching and $M$ (which we allow to depend on $\pi$ ) has at most $m$ nonzero entries. This is because we can ignore the all- 0 rows and columns, so the size of $M$ can be bounded, and then there are only finitely many such matrices, so $\mathcal{C}$ will lie in the grid class of their direct sum (which also has a matching for its graph). Equivalently, by our remarks above, it suffices to show that every permutation in $\mathcal{C}$ can be divided into a bounded number of uninterrupted monotone intervals.

To this end, take some permutation $\pi \in \mathcal{C}$ of length $n$ with $N$-gridding given by $1=c_{1} \leq \cdots \leq c_{t+1}=n+1$ and $1=r_{1} \leq \cdots \leq r_{u+1}=n+1$ and consider a particular block in this gridding, say

$$
\pi^{(k, \ell)}:=\pi\left(\left[c_{k}, c_{k+1}\right) \times\left[r_{\ell}, r_{\ell+1}\right)\right) .
$$

We consider four types of alternations that elements of this block can participate in: vertical alternations either with blocks of the form $\pi^{\left(k, \ell^{+}\right)}$for $\ell^{+}>\ell$ or of the form $\pi^{\left(k, \ell^{-}\right)}$ for $\ell^{-}<\ell$, and horizontal alternations with blocks of the form $\pi^{\left(k^{+}, \ell\right)}$ for $k^{+}>k$ or of the form $\pi^{\left(k^{-}, \ell\right)}$ for $k^{-}<k$.

Every time that two consecutive elements in a block are separated either horizontally or vertically (that is, every time that two consecutive elements in a block fail to lie in an uninterrupted monotone interval together), they contribute to the length of at least one of these four alternations. Therefore, at most $4 d$ such separations can occur, so $\pi^{(k, \ell)}$ can be divided into at most $4 d+1$ uninterrupted monotone intervals. Hence $\pi$ itself can be divided into at most $(4 d+1) t u$ uninterrupted monotone intervals, proving the proposition.

Having established that sub- $2^{n-1}$ griddable classes (and in particular, sub-Fibonacci classes) lie in grid classes of matchings, we now have another proof of the Fibonacci dichotomy:

Corollary 3.4. For every permutation class $\mathcal{C}$, one of the following occurs:

- $\left|\mathcal{C}_{n}\right| \geq F_{n}$ for all $n$, or

- $\mathcal{C}$ lies in the grid class of a matching and there is a polynomial $p(n)$ so that $\left|\mathcal{C}_{n}\right|=$ $p(n)$ for all sufficiently large $n$. 


\section{Concluding remarks}

Decidability. It is not hard to see that the hypotheses of our characterisation theorems are decidable from the basis of a finitely based class. For example, in order to determine if $\operatorname{Av}(B)$ contains arbitrarily long direct sums of 21 one needs only check if any element of $B$ lies in $\operatorname{Av}(231,312,321)$, which is the set of permutations that are contained in arbitrarily long direct sums of 21 . Thus we have the following result.

Corollary 4.1. Given a finite set of permutations $B$, it is decidable whether or not $\operatorname{Av}(B)$ is griddable.

Similar arguments show that polynomial enumeration is decidable for finitely based classes. One first needs to check whether the class is griddable and then decide whether the class contains arbitrarily long alternations.

Corollary 4.2. Given a finite set of permutations $B$, it is decidable whether or not $\left|\operatorname{Av}_{n}(B)\right|$ agrees with a polynomial for all sufficiently large $n$.

Finite bases. The decidability results above only apply to finitely based classes, however, it happens that permutation classes with polynomial enumeration must be finitely based. Because these classes lie in grid classes of matchings, they also lie in grid classes of $0 / \pm 1$ row vectors. Now one needs only to apply the result of Atkinson, Murphy, and Ruškuc [7] that every subclass of the grid class of a $0 / \pm 1$ row vector is finitely based.

Enumeration. While Corollary 3.4 characterises the sub-Fibonacci classes and shows that they have eventually polynomial enumeration, it does not address the issue of how one might find these formulas. This could presumably be settled by strengthening the results given here to obtain bounds (computable from the basis of $\mathcal{C}$ ) on the degree of the polynomial and the values of $n$ for which $\left|\mathcal{C}_{n}\right|$ agrees with this polynomial, but there are already three general methods which can be used to count these classes:

(1) Since permutation classes with polynomial growth lie in grid classes of matchings, they also lie in grid classes of $0 / \pm 1$ row vectors. One can therefore use Theorem 2.7 to enumerate them.

(2) Permutation classes with polynomial growth contain only finitely many "simple permutations", and so the results of Albert and Atkinson [1] apply to them.

(3) Permutation classes with polynomial growth correspond to regular languages via the insertion encoding of Albert, Linton, and Ruškuc [4], so this method can also be used.

Grid classes of permutation matrices. A characterisation of the permutation classes that lie in grid classes of permutation matrices (Atkinson's profile classes from [6]) can be proved by replacing "uninterrupted monotone intervals" with "uninterrupted increasing 
intervals" in the proof of Proposition 3.3. These are the classes that lie in grid classes of matchings and do not contain arbitrarily long decreasing permutations.

Classes with two basis elements. Albert, Atkinson, and Brignall [2] have recently studied doubleton-based classes with polynomial enumeration. They give a characterisation of the pairs of permutations $\beta_{1}$ and $\beta_{2}$ for which $\operatorname{Av}\left(\beta_{1}, \beta_{2}\right)$ has polynomial enumeration and give bounds on the degree of this polynomial.

Acknowledgment. We thank Nik Ruškuc and Bruce Sagan for their helpful comments.

\section{References}

[1] Albert, M. H., And Atkinson, M. D. Simple permutations and pattern restricted permutations. Discrete Math. 300, 1-3 (2005), 1-15.

[2] Albert, M. H., Atkinson, M. D., And Brignall, R. Permutation classes of polynomial growth. arXiv:math.CO/0603315.

[3] Albert, M. H., Atkinson, M. D., And RušKuc, N. Regular closed sets of permutations. Theoret. Comput. Sci. 306, 1-3 (2003), 85-100.

[4] Albert, M. H., Linton, S., And RušKuc, N. The insertion encoding of permutations. Electron. J. Combin. 12, 1 (2005), Research paper 47, 31 pp. (electronic).

[5] Atkinson, M. D. Permutations which are the union of an increasing and a decreasing subsequence. Electron. J. Combin. 5 (1998), Research paper 6, 13 pp. (electronic).

[6] Atkinson, M. D. Restricted permutations. Discrete Math. 195, 1-3 (1999), 27-38.

[7] Atkinson, M. D., Murphy, M. M., And Ruškuc, N. Partially well-ordered closed sets of permutations. Order 19, 2 (2002), 101-113.

[8] Balogh, J., Bollobás, B., And Weinreich, D. The speed of hereditary properties of graphs. J. Combin. Theory Ser. B 79, 2 (2000), 131-156.

[9] Erdős, P., And Szekeres, G. A combinatorial problem in geometry. Compos. Math. 2 (1935), 463-470.

[10] Földes, S., And Hammer, P. L. Split graphs. In Proceedings of the Eighth Southeastern Conference on Combinatorics, Graph Theory and Computing (Louisiana State Univ., Baton Rouge, La., 1977) (Winnipeg, Man., 1977), Utilitas Math., pp. 311-315. Congressus Numerantium, No. XIX.

[11] Kaiser, T., AND Klazar, M. On growth rates of closed permutation classes. Electron. J. Combin. 9, 2 (2002/03), Research paper 10, 20 pp. (electronic).

[12] Kézdy, A. E., Snevily, H. S., And Wang, C. Partitioning permutations into increasing and decreasing subsequences. J. Combin. Theory Ser. A 73, 2 (1996), $353-359$.

[13] Knuth, D. E. The art of computer programming. Vol. 1: Fundamental algorithms. Addison-Wesley Publishing Co., Reading, Mass., 1969. 
[14] Lakshmibai, V., And Sandhya, B. Criterion for smoothness of Schubert varieties in SL(n)/B. Proc. Indian Acad. Sci. Math. Sci. 100, 1 (1990), 45-52.

[15] Marcus, A., And Tardos, G. Excluded permutation matrices and the StanleyWilf conjecture. J. Combin. Theory Ser. A 107, 1 (2004), 153-160.

[16] Murphy, M. M., And Vatter, V. Profile classes and partial well-order for permutations. Electron. J. Combin. 9, 2 (2002/03), Research paper 17, 30 pp. (electronic).

[17] Pouzet, M., And ThiÉRy, N. M. Some relational structures with polynomial growth and their associated algebras. arXiv:math.C0/0601256.

[18] Scheinerman, E. R., And Zito, J. On the size of hereditary classes of graphs. J. Combin. Theory Ser. B 61, 1 (1994), 16-39.

[19] Stankova, Z. E. Forbidden subsequences. Discrete Math. 132, 1-3 (1994), 291-316.

[20] Stanley, R. P. Solution to problem E2546. Amer. Math. Monthly 83, 10 (1976), 813-814. 